\title{
Capillary kinetics of thin polymer films in permeable microcavities
}

\author{
Kahp Y. Suh ${ }^{\text {a) }}$ and Pilnam Kim \\ School of Mechanical and Aerospace Engineering, Seoul National University, Seoul, 151-742, Korea \\ Hong $\mathrm{H}$. Lee \\ School of Chemical Engineering, Seoul National University, Seoul, 151-742, Korea
}

(Received 11 February 2004; accepted 3 September 2004)

\begin{abstract}
We present a Poiseuille model that can explain the rate of capillary rise of thin polymer films in permeable microcavities. In comparison to the traditional Poiseuille formulation, two features of the system were considered: the permeable nature of the enclosure and the effect of thin polymer films that are confined to the substrate. The model predicts that the rate is inversely proportional to the channel width, contrary to what the original Poiseuille model predicts, and it is proportional to the initial film thickness, which the original model cannot account for. The modified model is in satisfactory agreement with experimental data. (C) 2004 American Institute of Physics.
\end{abstract}

[DOI: $10.1063 / 1.1810212]$

Capillarity is involved in many practical systems such as wetting and repellency of woven fibers, paper products, and porous solids, wicking, and cleaning action in detergent baths. ${ }^{1}$ Above all, the capillary flow of molten polymers has been extensively studied in plastics because the polymer melt is often accompanied by instabilities called "melt fracture" in extrusion process. ${ }^{2}$ At very high shear stress, polymer melt flows violate the classical no-slip hydrodynamic boundary condition at solid surfaces, and exhibit nonNewtonian and complex nonlinear dynamics, which often leads to the instabilities. ${ }^{3}$

However, the polymer melt can be regarded as a Newtonian fluid and the no-slip boundary condition can be applied if the shear rate is sufficiently small. ${ }^{4}$ A typical example is capillary force lithography (CFL), which has recently been developed for patterning polymers above their glass transition temperatures. ${ }^{5}$ When a patterned PDMS mold is placed on a polymer film spin-coated onto a substrate and heated above the glass transition temperature, capillarity forces the polymer melt into the void of the channel. In contrast to the "forced" capillary flow in the conventional extrusion process, the flow in this case is "spontaneous" without any external force. In this spontaneous flow, the rate of capillary rise is very small due to the high viscosity of polymer melt and the shear rate is accordingly small. A similar situation can be found in high temperature nanoimprint lithography if a negative mold is used. ${ }^{6}$

For a smooth, uniform (laminar) flow of a fluid in a narrow cylindrical tube, which is the classical capillary system, the Poiseuille equation can be used to relate the volumetric flow rate to various characteristics of the fluid and the capillary system. The volumetric flow rate, $d V / d t$, is given by $^{7}$

$$
d V / d t=\pi r^{4} P / 8 \eta z
$$

where $r$ is the radius of the tube, $\eta$ is the viscosity of the fluid, $z$ is the distance of fluid movement in the tube in time, $t$, and $P$ is the pressure drop across the distance $z$. In Eq. (1), the polymer melt is a Newtonian fluid and the no-slip boundary condition is used. In the original Poiseuille formulation,

\footnotetext{
a) Author to whom correspondence should be addressed; electronic mail: sky4u@snu.ac.kr
}

the pressure drop, $P$, is replaced by Laplace pressure $(=\gamma \cos \theta / L)$, which yields

$$
d z / d t=L \gamma \cos \theta / 8 \eta z,
$$

where $L$ is the half channel width, $\gamma$ is the surface tension of the polymer, and $\theta$ is the equilibrium contact angle at the three-phase contact line.

Our experiments revealed that the original Poiseuille formulation fails when it is applied to the capillary rise of thin polymer melt into a microcavity, as one would find in CFL. For one, the filling rate is not proportional to the channel width but rather inversely proportional to the width, which is a behavior exactly opposite to that predicted by Eq. (2). For another, the rate is strongly dependent on the film thickness, for which the original Poiseuille formulation cannot provide any information.

Although nanoscopic and microscopic patterning of surfaces is drawing considerable interest that involves capillarity, ${ }^{5,6,8}$ there has been no suitable model that can explain the rate of capillary rise of fluids under confined geometry on a micro- and nano-scale. In this letter, we develop a Poiseuille model to explain the capillary kinetics of polymer melt in microcavities and compare the model with experimental data.

We fabricated a PDMS (Sylgard 184, Dow Corning) mold that has a planar surface with recessed patterns by casting PDMS against a complementary relief structure prepared by photolithographic method. Line-and-space patterns $(1,2$, and $3 \mu \mathrm{m}$ ) with a step height of $550 \mathrm{~nm}$ were used in our experiment. The mold with the pattern was placed on the surface of a polymer layer spin-coated onto a silicon substrate and then heated well above the glass transition temperature of the polymer. For the polymer, we used commercial symmetric styrene-butadiene-styrene (SBS) block copolymer (molecular weight $=3 \times 10^{5}$, styrene fraction $=0.3$, and $T_{g}=36^{\circ} \mathrm{C}$ ). Silicon wafer that was used as the substrate was cleaned by ultrasonic treatment in trichloroethylene and methanol for $5 \mathrm{~min}$ each and dried in nitrogen. The film thickness was measured by elipsometry and controlled by changing the concentration of the polymer solution $(3-10 \mathrm{wt} \%)$ and the coating speed (3000$8000 \mathrm{rpm})$. To exclude the solvent effect, we dried each sample under vacuum for $3 \mathrm{~h}$. Atomic force microscopy (AFM) measurements were made with a Nanoscope IIIa 


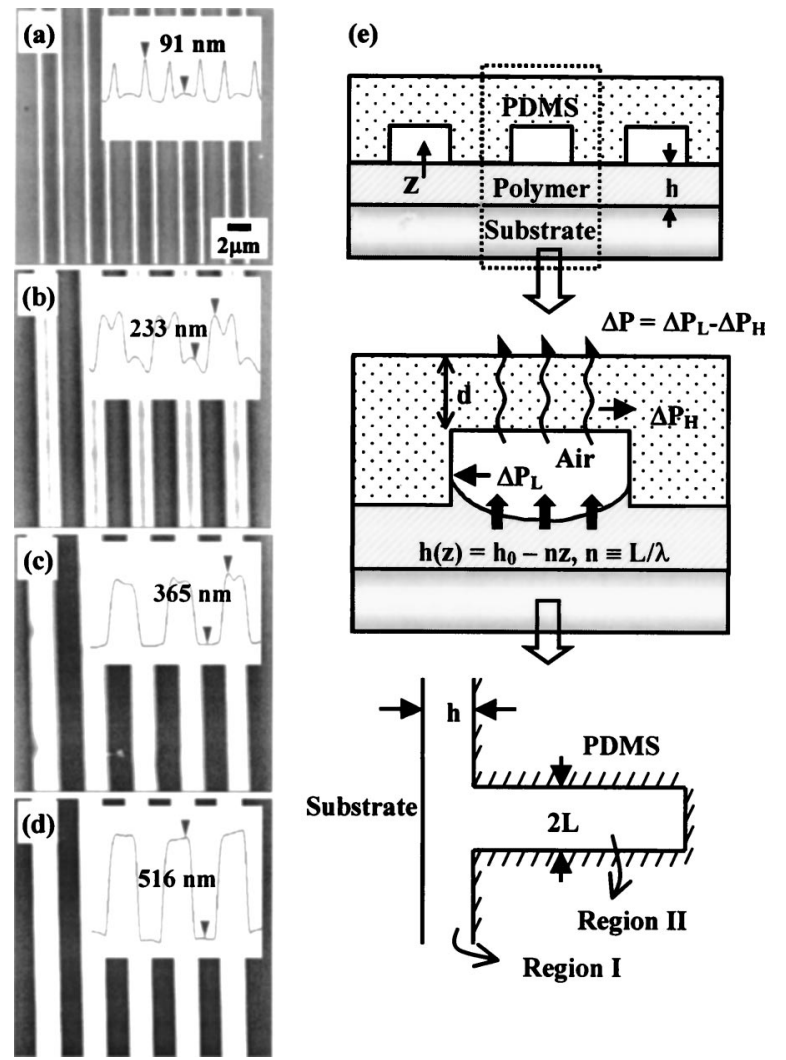

FIG. 1. (a)-(d) Planar and cross-sectional views of AFM profiles for the $2 \mu \mathrm{m}$ line-and-space pattern as a function of annealing time [(a) 10, (b) 30, (c) 60 , and (d) $180 \mathrm{~min}]$ at $70{ }^{\circ} \mathrm{C}$ for the film thickness of $1.0 \mu \mathrm{m}$. (e) Schematic illustration of the capillary rise into permeable microcavities and a series of channel resistances.

(Digital Instrument), operated in the contact mode. More information on the experimental details can be found elsewhere. 5

Figures 1(a) to 1(d) show the planar and cross-sectional views of AFM profiles for $2 \mu \mathrm{m}$ line-and-space pattern as a function of annealing time $(10,30,60$, and $180 \mathrm{~min})$ at $70{ }^{\circ} \mathrm{C}$ for the film thickness of about $1.0 \mu \mathrm{m}$. In the initial stage, the polymer film wets the wall to satisfy the equilibrium contact angle at the polymer/PDMS/air interface. From the cross-sectional AFM profile in Fig. 1(a), the contact angle can be calculated to be about $65^{\circ}$. The angle is given by the relationship, $\cos \theta=2(\Delta / L) /\left[1+(\Delta / L)^{2}\right]$ where $\Delta$ is the height difference between the highest and the lowest point of the meniscus and $L$ is the half channel width. If we use 20,6, and $30 \mathrm{~mJ} / \mathrm{m}^{2}$ for interfacial tensions at PDMSair, PDMS-SBS, and SBS-air interfaces, respectively, ${ }^{10}$ we obtain a contact angle of $62^{\circ}$, which is slightly smaller than the observed value.

As the mobility of the melt is very low compared with common liquids, it takes a considerable amount of time to form a uniformly curved meniscus within the channel. This incubation time is given by a complicated function of channel width, film thickness, surface tension, viscosity, and annealing temperature. ${ }^{11}$ Once the stable meniscus forms, the melt starts rising until it reaches the ceiling of the mold. It is noted here that the wetting angle should be realized first before the capillary rise takes place.

To gain an understanding of the capillary rise for our system, we consider a series of Poiseuille flows as illustrated in Fig. 1(e). The first flow occurs in the mass reservoir confined by the mold and substrate (region 1) while the second Downloaded 27 Sep 2005 to 147.46.242.161. Redistribution subject

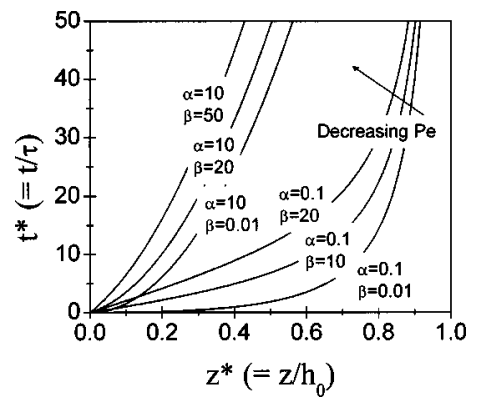

FIG. 2. Effect of permeability and film thickness on the rate of capillary rise for several values of $\alpha$ (aspect ratio) and $\beta$ (permeability parameter).

flow takes place in the polymer melt within the channels (region II). The channels are microcavities surrounded by PDMS walls through which the trapped air can permeate out of the mold. With the assumption of Poiseuille flow between two parallel plates, we can obtain the moving rate of polymer melt based on a series of channel resistances, which is given by

$$
\frac{d z}{d t}=\frac{\Delta P}{3 \eta z\left(1 / L^{2}+2 / h^{2}\right)},
$$

where $h$ is the time-dependent film thickness and the other parameters are defined in Fig. 1(e). Equation (3) reduces to the original Poiseuille equation if the film thickness is sufficiently large compared with the channel width. Since the mass transport is almost entirely from the adjacent regions and not from outside the mold, $h(z)$ is given by

$$
h(z)=h_{0}-n z, \quad n \equiv L / \lambda,
$$

where $h_{0}$ is the initial film thickness and $\lambda$ is half the space width. For $n \ll 1, h(z)$ is almost equal to $h_{0}$ during the capillary rise and the effect of film thickness disappears, which can readily be understood from mass conservation.

The driving force for the capillary rise $(\Delta P)$ is a little complicated since the mold is end-capped with permeable solid. As such, air can be trapped in the void space or the cavity such that the permeation kinetics of air should also be taken into consideration to accurately describe the rate of capillary rise. Therefore, the net driving force is given by the difference of two pressure drops, which is
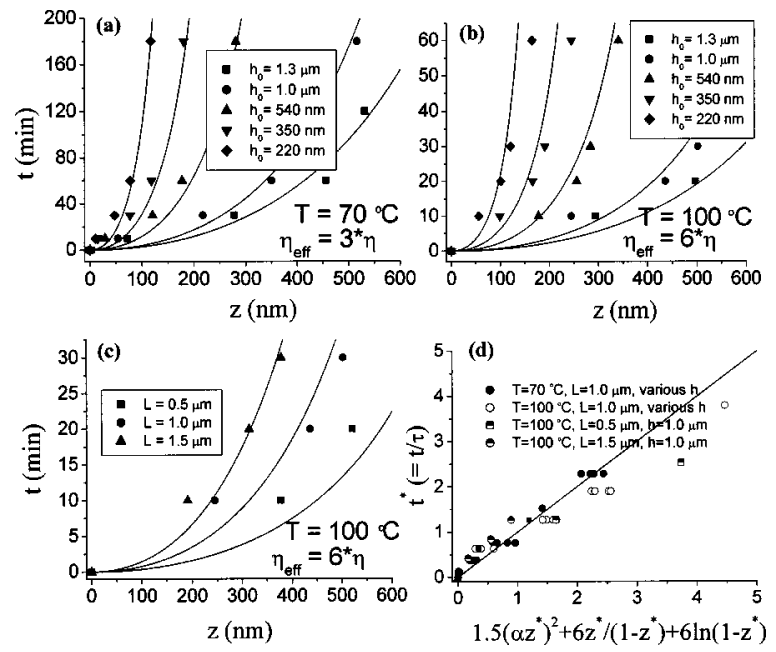

FIG. 3. Comparison of the theoretical model with the experimental data at (a) $70{ }^{\circ} \mathrm{C}$ and (b) $100{ }^{\circ} \mathrm{C}$ for various film thicknesses for the strip width of $2 \mu \mathrm{m}(L=1 \mu \mathrm{m})$. (c) Effect of channel width on the rate of capillary rise at

$100{ }^{\circ} \mathrm{C}$ for the film thickness of $1.0 \mu \mathrm{m}$. (d) A generalized diagram as a function of the dimensionless time and length.

to AIP license or copyright, see http://apl.aip.org/apl/copyright.jsp 


$$
\Delta P=\Delta P_{L}-\Delta P_{H},
$$

where $\Delta P_{L}$ is the Laplace pressure and $\Delta P_{H}$ is the difference in hydrostatic pressure between the cavity and outside the mold. From the definition of permeability, Pe, and the assumption of one-dimensional steady-state penetrant flux through the mold, $\Delta P_{H}$ can be written as ${ }^{12}$

$$
\Delta P_{H}=\frac{d}{\operatorname{Pe}} \frac{d z}{d t},
$$

where $d$ is the permeation length of the mold in Fig. 1(e). Inserting Eqs. (5) and (6) into Eq. (3) leads to the following kinetic equation:

$$
\frac{d L^{2}}{3 \eta \mathrm{Pe}} z+\frac{1}{2} z^{2}+\frac{2 z}{h_{0}-z} L^{2}-2 \ln \left(\frac{h_{0}}{h_{0}-z}\right) L^{2}=\frac{L \gamma \cos \theta}{3 \eta} t
$$

or in a dimensionless form,

$$
\beta z^{*}+\frac{3}{2}\left(\alpha z^{*}\right)^{2}+\frac{6 z^{*}}{1-z^{*}}+6 \ln \left(1-z^{*}\right)=t^{*}
$$

where $z^{*} \equiv z / h_{0}, \quad t^{*} \equiv t / \tau, \quad \alpha \equiv h_{0} / L \quad$ (aspect ratio), $\beta$ $\equiv(d / \mathrm{Pe}) /\left(\eta / h_{0}\right)$ (permeation resistance/viscous resistance), $\tau \equiv(\eta L / \gamma \cos \theta)=\left(\eta / \Delta P_{\mathrm{L}}\right)$, and $n$ is assumed to be unity for convenience (equal line and space). The physical meaning of $\tau$ can be interpreted as a time scale for the capillary rise, which is proportional to viscosity and inversely proportional to Laplace pressure.

To examine the effect of permeability and film thickness on the capillary kinetics, Eq. (8) is plotted in Fig. 2 for several values of $\alpha$ and $\beta$. As shown in the figure, one can see the transition from a linear to nonlinear growth as the permeability increases. The permeability of air in PDMS was reported to be about $3.6 \times 10^{-2} \mu \mathrm{m}^{2} / \mathrm{Pa}$ s at $35^{\circ} \mathrm{C} .{ }^{12}$ It decreases slightly with increasing temperature. If we insert typical values of $d(\sim 0.5 \mathrm{~cm}), \eta\left(\sim 10^{6} \mathrm{~Pa}\right.$ s at $100{ }^{\circ} \mathrm{C}$ from our rheometrics spectroscopy measurement), and $L(\sim 1.0 \mu \mathrm{m})$, and the maximum value of $h_{0}(\sim 1.3 \mu \mathrm{m})$, the maximum value of $\beta$ is about $1.8 \times 10^{-2}$. Thus, the contribution of gas permeation to the overall kinetics is negligible for PDMS stamps, which is in part due to the fact that PDMS is presumably the most permeable rubbery polymer for $\mathrm{O}_{2}$ and $\mathrm{N}_{2}$. ${ }^{13}$

Here, we investigate three limiting cases of Eq. (7). The first is one in which the mold is highly permeable and the film thickness is very small. Then, Eq. (7) reduces to

$$
\frac{z}{h_{0}-z}-\ln \left(\frac{h_{0}}{h_{0}-z}\right)=\frac{\gamma \cos \theta}{6 \eta L} t .
$$

In this case, the capillary rise $z$, or the distance the polymer melt covers, increases nonlinearly with time, in contrast to the relationship $z \propto t^{1 / 2}$ in the original Poiseuille equation. Furthermore, the rate is inversely proportional to the channel width $L$. This is readily understood in that the channel resistance in region I is dominant over that of the Poiseuille flow in region II. A typical example for this situation can be found in CFL as long as the film thickness is relatively small. ${ }^{5}$

The second limiting case arises when the gas permeation is sufficiently slow and Eq. (7) reduces to

$$
z=\frac{\operatorname{Pe} \gamma \cos \theta}{d L} t
$$

In this case, the capillary rise increases linearly with time. In addition, the contribution of viscosity and film thickness disappears such that the kinetics is solely governed by the permeation kinetics and capillary force. A typical example for this situation can be found in nanoimprint lithography if negative molds are used. ${ }^{6}$

The third is one in which the mold is highly permeable and the film thickness is sufficiently large and the equation reduces to

$$
z=\left(\frac{2 L \gamma \cos \theta}{3 \eta} t\right)^{1 / 2}
$$

which corresponds to the original Poiseuille equation. It was reported that Eq. (11) qualitatively agrees with the rate of capillary flow in micromolding in capillaries. ${ }^{9}$ Thus, the general kinetic equation derived in Eq. (7) or (8) can be applied to various capillary systems reported in the literature.

In Fig. 3, we compared the theoretical model with the experimental data at (a) $70{ }^{\circ} \mathrm{C}$ and (b) $100{ }^{\circ} \mathrm{C}$ for various film thicknesses for the strip width of $2 \mu \mathrm{m}(L=1 \mu \mathrm{m})$. For convenience, we assume that the average height of the meniscus accounts for the capillary rise during the formation of a uniform curvature within the channel. The rate of capillary rise strongly depends on several extrinsic factors such as wetting condition of the mold, resulting in a scattering of the data. We also compared the kinetic equation for various channel widths for a given thickness of $1.0 \mu \mathrm{m}$ in Fig. 3(c), which is in reasonable agreement with the data. A generalized diagram shown in Fig. 3(d) shows the validity of the kinetic equation for various film thicknesses and channel widths. The fitted viscosity of the film ( $\left.\eta_{\text {eff }}\right)$ is slightly larger than the bulk value by three to six times depending on the temperature tested, which might be attributed to unsteady capillary flow and confinement effects.

In summary, we developed a simple and yet versatile Poiseuille equation to explain the kinetics of capillary rise of the polymer melt into permeable microcavities. It is found that the rate of capillary rise is proportional to the film thickness and also to the degree of wetting but inversely proportional to the viscosity and the channel width. This simple kinetics could provide valuable tools for predicting the behavior of polymer melts under various capillary systems.

This work was supported by the Brain Korea 21 Project in 2004.

${ }^{1}$ A. W. Adamson and A. P. Gast, Physical Chemistry of Surfaces, 6th ed. (Wiley, New York, 1997).

${ }^{2}$ A. I. Leonov and A. N. Prokunin, Nonlinear Phenomena in Flows of Viscoelastic Polymer Fluids (Chapman and Hall, London, 1994).

${ }^{3}$ W. B. Black and M. D. Graham, Phys. Rev. Lett. 77, 956 (1996).

${ }^{4}$ J. D. Ferry, Viscoelastic Properties of Polymers, 3rd ed. (Wiley, New York, 1980).

${ }^{5}$ K. Y. Suh, Y. S. Kim, and H. H. Lee, Adv. Mater. (Weinheim, Ger.) 13, 1386 (2001).

${ }^{6}$ H.-C. Scheer, H. Schulz, T. Hoffmann, and C. M. Sotomayor Torres, J. Vac. Sci. Technol. B 16, 3917 (1998).

${ }^{7}$ D. Myers, Surfaces, Interfaces, and Colloids (VCH, New York, 1991), pp. 87-109.

${ }^{8}$ S. Y. Chou, P. R. Krauss, and P. J. Renstrom, Science 272, 85 (1996).

${ }^{9}$ E. Kim, Y. Xia, and G. M. Whitesides, Nature (London) 376, 581 (1995).

${ }^{10}$ J. Brandrup and E. H. Immergut, Polymer Handbook (Wiley, New York, 1989).

${ }^{11}$ K. Y. Suh, S. Chou, and H. H. Lee, J. Micromech. Microeng. 14, 1185 (2004).

${ }^{12}$ T. C. Merkel, V. I. Bondar, K. Nagai, B. D. Freeman, and I. Pinnau, J. Polym. Sci., Part B: Polym. Phys. 38, 415 (2000).

${ }^{13}$ B. D. Freeman and I. Pinnau, Trends Polym. Sci. 5, 167 (1997) 